Gradienten bestimmter Faktoren zur Mutantenauslese und -Anreicherung, führen.

Summary. Using $\mathrm{CO}_{2}$ in manometric control of continuous cultures (Sacch. cer.), the cultures were maintained constant in intensity of metabolism in respect to $\mathrm{CO}_{2}$ production. The small fermentors $(50 \mathrm{ml})$ are useful for investigations in metabolism under various conditions at a steady state.

Institut für Mikrobiologie und Weinforschung

K. KLEMM

der Johannes Gutenberg-Universität,

Ernst-Ludwig-Strasse 10,D-6500 Mainz (Germany),

9 March 1972.

\title{
Ein einfacher Impulssummen-Schreiber zur digitalen Registrierung von Prozessabläufen
}

Der angegebene Schreiber registriert und summiert Impulse in der Zeit und eignet sich damit zur Aufzeichnung von Prozessabläufen in digitaler Form. Einzige Voraussetzung für den Einsatz ist die Möglichkeit der Übernahme definierter Schaltimpulse aus dem interessierenden Ablauf. Das Gerät wurde zur zeitlichen Überwachung einer manometrisch geregelten, kontinuierlichen Fermenteranlage entworfen und gebaut ${ }^{1}$. Auch zur Verfolgung des Gärverlaufes in Standkulturen anhand der $\mathrm{CO}_{2}-$ Bildung wurde es mit Erfolg eingesetzt.

Das Gerät unterscheidet sich von handelsüblichen Schreibern Jurch die vereinfachte Aufzeichnung in der Ordinate. Der Zeitvorschub in der Abszisse erfolgt in üblicher Form, entweder durch zeitlich konstanten $\mathrm{Pa}$ piervorschub oder Bewegung einer Brücke mit dem Ordinlatenantrieb über das festliegende Registrierpapier.

Zur Registrierung in der Ordinate sitzt die Schreibspitze an einer Spindel, deren Antriebsmotor über ein Relais durch die $z u$ registrierenden Impulse angesteuert wird. Die Vorschubstrecke pro Impuls kann durch Wahl der Spindelsteigerung oder des Antriebsmotors dem Verwendungszweck angepasst werden. Der Einsatz eines Schrittmotors dürfte dabei die zweckmässigste Lösung darstellen.

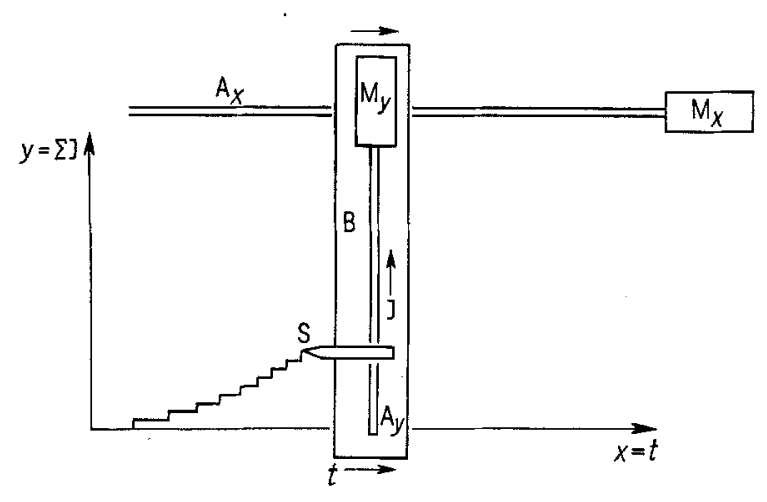

Bei der verwendeten Ausführung (Figur) wird entlang der Abszisse als Zeitachse eine Brücke (B) mit konstanter Geschwindigkeit über das Registrierpapier geführt (normales Millimeterpapier). Die Vorschubgeschwindigkeit ergibt sich aus der Steigung der Spindel $A_{x}$ (10 mm) und der Drehzahl des Getriebemotors $M_{x}(1 \mathrm{U} / \mathrm{h})$ zu $10 \mathrm{~mm} / \mathrm{h}$. Zur Impulsaufzeichnung in der Ordinate sitzt die Schreibspitze S, eine Bleistiftmine, an der Spindel $A_{y}$ (1,5 mm Steigung) auf der Brücke B. Der Antrieb erfolgt durch einen Getriebemotor $M_{y}(2 \mathrm{U} / \mathrm{min})$. der über ein Zeitrelais $(6 \mathrm{sec})$ angesteuert wird, d.h. die Vorschubstrecke pro Impuls beträgt bei dieser Ausführung $0,3 \mathrm{~mm}$ pro Impuls.

Da sich die Schreibspitze ohne Rücklauf bewegt, werden die Impulse in Form einer Treppenkurve registriert und damit in der Ordinate geometrisch summiert. Bei kleinen Schreiberbewegungen (wie oben angegeben und kleiner) ist die Treppenkurve als solche nur mit der Lupe deutlich zu erkennen. In einem solchen Fall kann sich die Zuschaltung eines Impulszählwerkes als zweckmässig erweisen.

Sind die Schaltimpulse durch Auslösung stets quantitativ gleicher Reaktionen auf den Prozessverlauf definiert, so ist die Summe der Impulse proportional der Summe dieser Reaktionen. Im Falle der angegebenen Fermenterüberwachung erfolgt je Schaltimpuls eine quantitativ gleiche Abgabe von $\mathrm{CO}_{2}$ wie auch $\mathrm{Zu}$ - und Abgabe von Substrat. In einem solchen Fall können diese Werte anhand der Impulssumme auf der Ordinate direkt abgelesen werden. Der Anstieg der Registrierkurve ist dabei grundsätzlich ein Mass für die Geschwindigkeit des Prozessablaufes, bei dem oben angegebenen Fermenter für die Stoffwechselaktivität der gärenden Hefen.

Ein Einbau verschiebbarer Schalter an den Achsenantrieben gibt die Möglichkeit, zusätzliche Schaltimpulse nach vorgewählter Zeit oder Erreichen einer bestimmten Impulszahl auszulösen. Sind gegenläufige Vorgänge zu registrieren, etwa eine $\mathrm{Ph}-$ Kontrolle durch sowohl Säurewie Basenzugabe, kann entweder ein Motor mit umkehrbarer Drehrichtung oder ein Planetengetriebe mit gegenläufigem Gehäuseantrieb für die Ordinatenbewegung verwendet werden.

Die Verwendung von Relais zur Ansteuerung in der Ordinate erübrigt eine elektrische Anpassung. Das Gerät ist generell zur digitalen Registrierung geeignet, es muss lediglich die Möglichkeit gegeben sein, dem interessierenden Prozessablauf definierte Schaltimpulse zu entnehmen.

Summary. The coordinate plotter summarizes impulses from digital control mechanisms geometrically in terms of time. It is possible to obtain additional impulses from the plotter by mounting of special switches.

K. KLEMM

Institut für Mikrobiologie und Weinforschung der Johannes Gutenberg-Universität, Emst-Ludwig-Str. 10 D-6500 Mainz (Germany), 9. März 1972.

\footnotetext{
1 K. KLEMM, Experientia 28, 1527 (1972).
} 